\title{
Herpes simplex virus Type 2 and other genital ulcerative infections as a risk factor for $\mathrm{HIV}-1$ acquisition
}

Ireneus P M Keet, Francis K Lee, Godfried J P van Griensven, Joep M A Lange, André Nahmias, Roel A Coutinho

\begin{abstract}
We studied the role of genital ulcerative infections for acquisition of human immunodeficiency virus type 1 (HIV-1) infection in a cohort of 989 homosexual men in Amsterdam between October 1984 and December 1988. Among 53 HIV-1 seroconverters serological and anamnestic data were gathered regarding herpes simplex virus type 2 (HSV-2) and syphilis in the 6 months before seroconversion. For statistical analysis a control who remained seronegative during the same interval was selected at random for each HIV-1 seroconverter. A significant difference between the prevalence of HSV-2 antibodies among HIV-1 seroconverters and controls was found $(72 \%$ vs $38 \%$ ). HSV-2 seroconversions among men initially seronegative for $\mathrm{HSV}-2$ were found among three of $18 \mathrm{HIV}-1$ seroconverters and among three of 36 controls. (O.R. $=2 \cdot 2,95 \%$

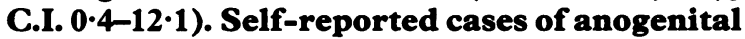
herpes were found more frequently among HIV-1 seroconverters (8) than among controls (4). One case of syphilis was diagnosed among HIV-1 seroconverters, and one among controls. Summing up these cases we assessed the total number of genital ulcerative infections: 12 among HIV-1 seroconverters and eight among controls (23 vs $15 \%$, O.R. 1.7, C.I. 0.64.62). These data suggest little evidence for
\end{abstract}

Department of Infectious Diseases, Municipal Health Service, PO Box 20244, 1000 HE Amsterdam, The Netherlands

Ireneus P M Keet, Godfried J P van Griensven, Roel A Coutinho

Division of Infectious Diseases and Immunology, Department of Pediatrics, Emory University School of Medicine, Atlanta, USA

Francis K Lee, André Nahmias

Department of Virology, University of Amsterdam, The Netherlands

Joep M A Lange genital ulcerative infections being an important independent risk factor for HIV-1 acquisition among homosexual men in Amsterdam during the time period studied.

Data from two recent studies suggest that anogenital ulcerative diseases such as herpes simplex virus type 2 (HSV-2) and syphilis may be important in the acquisition of human immunodeficiency virus type 1 (HIV-1) infection in homosexual men. ${ }^{12}$

This relation is plausible for two reasons. First the lesions may provide a portal of entry for HIV-1, thereby enhancing transmission. Secondly the inflammatory response may increase the number of activated T-lymphocytes at the site of these ulcerations, resulting in a rise in susceptibility for HIV-1 infection. ${ }^{3}$ Several epidemiological studies in Africa support this association among heterosexuals. ${ }^{45}$ Among homosexual men however there are few epidemiological data to support the hypothesis that anogenital ulcerative disease is an independent risk factor.

Several studies among homosexual men have demonstrated an association between HIV-1 infection and a history of syphilis, serological evidence of syphilis, a history of anogenital herpes and antibody to HSV-2. ${ }^{12}$ After correction for the number of sexual partners Stam et al found this association still to be significant. ${ }^{2}$ Only in one study was the relation in time studied between HIV-1 and HSV-2 seroconversion, in which was found that HIV-1 seroconverters significantly more often had a concurrent or preceding HSV-2 seroconversion then men from the same cohort who remained HIV-1 seronegative. ${ }^{1}$ This finding was still significant after correction for age, number of lifetime sexual partners and percentage of sexual acts involving sexual intercourse.

In our present study we investigated whether this association was also found among 989 homosexual men, prospectively followed in the Amsterdam cohort study between October 1984 and December 1988. We studied the prevalence and incidence of syphilis and HSV-2 infection among HIV-1 seroconverters and compared this with controls. who 
remained HIV-1 seronegative during the same time interval.

\section{Study population}

In the Amsterdam cohort study 989 homosexual men were entered after October 1984, of whom 236 men were seropositive for antibodies to HIV-1 at entry. These men are seen at 3 monthly intervals at the Municipal Health Service. During each visit a standardised full medical history was taken, a questionnaire regarding sexual behaviour was completed and bloodsamples are drawn for the presence of HIV-1 antibodies and syphilis serology. A routine part of the medical history was the question whether the participant noticed an anogenital herpes infection in the previous 3 months.

During follow-up $78 \mathrm{HIV}-1$ seroconversions were registered among $753 \mathrm{HIV}-1$ seronegatives. We selected the 54 men who had seroconverted from HIV-1 negative to positive after follow up of at least 6 months and had visited the Municipal Health Service at least twice before HIV-1 seroconversion.

We studied the incidence of genital ulcerative infections in the six months prior to HIV-1 seroconversion. The number of $\mathrm{HSV}-2$ seroconversions was studied by serological testing of stored sera, the number of self reported cases of anogenital herpes was retrieved from the medical history data and cases of syphilis were diagnosed on the basis of serology, darkfield microscopy and clinical data. For each HIV-1 seroconverter we randomly identified a man from the same cohort who had serum samples drawn in the same months, but who remained HIV-1 seronegative. Specimen 2 of these seronegative controls was drawn within a time-interval of 1 month before or after specimen 2 of the HIV-1 seroconverters. Of the HIV-1 seroconversions in this study seven took place in 1985, 25 in 1986, 14 in 1987 and eight in 1988. To control for factors known to be important for HIV-1 infection we retrieved information about age, number of life-time sexual partners and number of sexual partners during the 6 months prior to seroconversion.

\section{Laboratory methods}

Presence of HIV-1 antibodies was demonstrated by two commercially available enzyme linked immunosorbent assays (ELISA, Abbot Laboratories, North Chicago, Ill, USA; Vironostika Teknika Organon, Oss, The Netherlands). Seropositivity was confirmed by immunoblotting.

Serological evidence for syphilis was demonstrated by Treponema pallidum haemagglutination assay (TPHA) and Veneral Disease Research Laboratory (VDRL), both performed in the Laboratory of Public Health of the Amsterdam
Municipal Health Service (head: Dr G J van Doornum). In case of a fourfold (or more) rise of VDRL titre or TPHA seroconversion participants were seen again for repeated serology and-if applicabledarkfield microscopy. Stored specimens were analysed blindly for the presence of antibodies to HSV-1 and 2. Enzyme linked immunodot serologic assays were used to detect and differentiate HSV-1 and HSV-2 specific antibodies. Type-specific glycoproteins $\mathrm{gG}-1$ and gG-2 served as antigen for the assay. ${ }^{67}$ From each HIV-1 seroconverter two stored sera were analysed: specimen 1, drawn 6-9 months prior to HIV-1 seroconversion and specimen 2 , the first serum positive for HIV-1 antibodies. From each control two sera were analysed as well, matched in time with specimen 1 and 2 of the HIV-1 seroconverters.

\section{Statistical methods}

Statistical comparisons between cases and controls regarding categorial variables were made with test of proportion, utilising $\chi^{2}$ and Fisher exact-test. Age and the number of partners were compared utilising the $t$ test and the rank sum test (Wilcoxon test).

\section{Results}

\section{General characteristics}

Among the 78 HIV-1 seroconverters 54 met the selection criteria. Stored sera were available of 53 of the selected HIV-1 seroconverters and of all 54 controls. We compared these groups for the number of sexual partners, both lifetime and in the 6 months prior to HIV-1 seroconversion. Rank sum tests show that both groups are similar for these characteristics. The mean age for HIV-1 seroconverters and controls was 35 and 36 years respectively (table 1 ).

\section{HSV serology}

Table 2 shows an association between HIV-1 seroconversion and prevalence of HSV-2 antibodies. In specimen 2 , taken at the moment of seroconversion, $38(71 \%)$ of 53 HIV-1 seroconverters are seropositive for HSV-2 antibodies and only $21(38 \%)$ of 54 controls $\left(\chi^{2} \mathrm{p}<0.05\right)$. Conversion to HSV-2 seropositivity between specimen 1 and 2 was found in three $(17 \%)$ of the $18 \mathrm{HIV}-1$ seroconverters and in three $(8 \%)$ of 36 controls (O.R. $=2 \cdot 2,95 \%$ C.I. $0 \cdot 4$ $12 \cdot 1)$.

Table 2 shows no difference in HSV-1 seroprevalence between HIV-1 seroconverters and controls. For both groups $72 \%$ of specimen 2 is seropositive for HSV-1. Seroconversion to HSV-1 positivity was found in two HIV-1 seroconverters and in one control.

Self reported cases of anogenital herpes

We studied the number of self-reported cases of 
Table 1 Characteristics of 53 HIV-1 seroconverters and 54 controls in the Amsterdam cohort study between October 1984 and December 1988

\begin{tabular}{|c|c|c|c|c|c|}
\hline & \multicolumn{2}{|c|}{ Seroconverters $(n=53)$} & \multicolumn{2}{|c|}{ Controls $(n=54)$} & \multirow[b]{2}{*}{$p$} \\
\hline & $M^{\star}$ & $S D \dagger$ & $M$ & $S D$ & \\
\hline $\begin{array}{l}\text { Age at enrollment } \\
\text { No of sexual partners during lifetime } \\
\text { No of sexual partners during last six months } \S\end{array}$ & $\begin{array}{r}35 \\
799 \\
14\end{array}$ & $\begin{array}{r}7 \\
2028 \\
18\end{array}$ & $\begin{array}{r}36 \\
866 \\
15\end{array}$ & $\begin{array}{r}8 \\
1553 \\
30\end{array}$ & $\begin{array}{l}\text { nsł } \\
\text { ns } \\
\text { ns }\end{array}$ \\
\hline
\end{tabular}

$\star M:$ mean.

+SD: standard deviation.

†ns: not significant.

$\S$ No of partners according to questionnaire completed at the visit when specimen 2 was taken.

Table 2 Comparison of serological results of herpes virus type $2(H S V-2)$ and type 1 (HSV-1) tested in HIV-1 seroconverters and controls in the Amsterdam cohort study between October 1984 and December 1988

\begin{tabular}{lcc}
\hline & Seroconverters $(n=53)$ & Controls $(n=54)$ \\
\hline HSV-2 tested positive in specimen $1(\%)$ & $35 / 53(66)$ & $18 / 54(33)$ \\
HSV-2 seroconversions $(\%) / H S V-2$ tested negative in specimen 1 & $3 / 18(17)$ & $3 / 36(8)$ \\
HSV-1 tested positive in specimen $1(\%)$ & $36 / 53(68)$ & $38 / 54(70)$ \\
HSV-1 seroconversions $(\%) / H S V-1$ tested negative in specimen 1 & $2 / 17(12)$ & $1 / 16(6)$ \\
\hline
\end{tabular}

anogenital herpes in the period between specimen 1 and 2 . Of the $35 \mathrm{HIV}-1$ seroconverters positive for HSV-2 antibodies in specimen 1 eight men reported an episode of anogenital herpes in the period between specimen 1 and $2(22 \%)$. Of the 18 controls positive for HSV-2 antibodies in specimen 1 four men reported an episode of anogenital herpes in the same period $(22 \%)$. None of the participants who were HSV-2 seronegative in specimen 1 , including the six who had serological evidence of a primary HSV-2 infection in specimen 2 , reported a history of anogenital herpes during the studied period.

\section{Syphilis}

In the interval between specimen 1 and 2 two cases of syphilis-I were diagnosed in the examined population, both reinfections in TPHA-positive men, one among the HIV-1 seroconverters and one among the controls.

Genital ulcerative infections and $H I V-1$ seroconversion In this study we collected data on three types of genital ulcerative infections: primary $\mathrm{HSV}-2$ infection, recurrent anogenital herpes infection and syphilis. Ulcerations of other aetiology were not reported. If we consider them to be one group of genital ulcerative disease, we find that HIV-1 seroconversions are preceded by either syphilis, HSV-2 seroconversion or recurrent anogenital herpes infection in 12 of 53 men $(22 \%)$. Of the $54 \mathrm{HIV}-1$ seronegative controls eight men $(15 \%)$ had a genital ulcerative infection during the same period (O.R. = $1 \cdot 7,95 \%$ C.I. $0 \cdot 63-4 \cdot 62$ ).

\section{Discussion}

Our sero-epidemiolgical study shows little evidence that anogenital ulcerative infection is a risk factor for HIV-1 acquisition among homosexual men. Apparently the relative risk of $\mathrm{HSV}-2$ primary infections (O.R. $=2 \cdot 2)$ and genital ulcerative infections in general $(O . R .=1 \cdot 7)$ is small. We found a strong association between $\mathrm{HSV}-2$ seropositivity and HIV-1 seroconversion, an association also found in other studies. This finding does not provide sufficient evidence as sexual behaviour in the past is a confounder.

It appears that in the studied period the transmission rate of HSV-2 is low; only six seroconversions were found. This can partially be explained by a saturation effect for HSV-2 infection as in our study $66^{\circ} \%$ of the HIV-1 seroconverters are already HSV-2 seropositive before HIV-1 seroconversion. In a comparable study by Holmberg et al this was only $45 \%$. $^{1}$

The 20 self-reported cases of anogenital herpes in this study were only found among HSV-2 seropositive men; we assume they were all recurrences. No HSV-2 seroconversion coincided with a selfreported case. This does not mean that these were all asymptomatic infections. An unknown percentage of HSV-2 infections is "unrecognised symptomatic". 89 It is plausible that this percentage is higher for primary infections, as in that case the person never experienced the symptoms before.

We found that $12 \mathrm{HIV}-1$ seroconverters and eight controls had a history of anogenital herpes infection during the studied time interval. For both groups this is $22 \%$ of the HSV-2 seropositives, the higher 
number of cases among HIV-1 seroconverters therefore corresponds with the prevalence of HSV-2 antibodies. Recurrence of HSV-2 infection is a common finding, a rate of 0.33 per month has been reported. ${ }^{10}$ Because of the location $\mathrm{HSV}-2$ infections among homosexual men are less frequently noticed by the infected individual than among heterosexuals. We must therefore consider the possibility that men with serological evidence of HSV-2 infection could have had recurrent episodes of anogenital ulceration due to herpes that were unapparent, which could result in an underestimation of the relative risk of acquiring $\mathrm{HIV}-1$ infection. There was no difference in the incidence of syphilis, in each group one case was diagnosed.

Our finding contrasts with the previously mentioned study of Holmberg et al who demonstrated a much higher relative risk of $\mathrm{HSV}-2$ seroconversions for HIV-1 acquisition among homosexual men. This study however was conducted among samples gathered in an earlier phase of the AIDS epidemic, when there was a higher level of sexual activity and HSV-2 seroconversions were seen frequently. The HIV-1 infections in our study occurred in a later phase when a radical change in sexual behaviour took place together with a strong decline of syphilis incidence. ${ }^{1112} \mathrm{We}$ conclude that genital ulcerative disease has not been a major risk factor for HIV-1 acquisition among homosexual men in Amsterdam in the years 1985-1988.

We thank the participants in this study; $M$ Bakker, $N$ Albrecht-van Lent, JM Nittel, P Krijnen, JW Mulder and PJ Veugelers for their help.

This study was supported by grants 28-1127 and 281026 from the Netherlands Foundation for Preventive Medicine.
Address for correspondence: IPM Keet, MD Department of Infectious Diseases, Municipal Health Service, PO Box 20244, 1000 HE Amsterdam, The Netherlands.

1 Holmberg SD, Stewart JA, Russel Gerber A, et al. Prior herpes simplex virus type II infection as a risk factor for HIVinfection. $J A M A$ 1988;259:1048-50.

2 Stamm WE, Hunter Handsfield H, Rompalo AM, Ashley RL, Roberts PL, Corey L. The association between genital ulcer disease and acquisition of HIV infection in homosexual men. JAMA 1988;260:1429-33.

3 Lukehart SA, Baker-Zander SA, Lloyd RM, et al. Characterisation of lymphocyte responsiveness in early experimental syphilis. J Immunol 1980;123:461-7.

4 Cameron DW, Simonsen JN, D'Costa LJD, et al. Female to male transmission of Human Immunodeficiency Virus type 1: Risk factors for seroconversions in men. Lancet 1989;334: 403-7.

5 Kreiss JK, Koech D, Plummer FA, et al. AIDS virus infections in Nairobi prostitutes: spread of the epidemic to East Africa. N Engl J Med 1986;314:414-8.

6 Lee FK, Coleman RM, Pereira L, Bailey P, Tatsuno M, Nahmias AJ. Detection of herpes simplex virus type 2-specific antibody with glycoprotein G. J Clin Microbiol 1985;22: $641-4$.

7 Lee FK, Pereira L, Griffin C, Reid E, Nahmias A. A novel glycoprotein for detection of herpes simplex virus type 1specific antibodies. $J$ Virol Meth 1986;14:111-8.

8 Mertz GJ, Schmidt O, Jourden JL, et al. Frequency of first episode genital infection with herpes simplex virus from symptomatic and asymptomatic source contacts. Sex Trans Dis 1985;12:33-9.

9 Reeves W.C, Corey L, Adams HG, Vontver LA, Holmes KK. Risk of recurrence after first episodes of genital herpes. $N$ Engl J Med 1981;305:315-9.

10 Lafferty WE, Coombs RW, Benedetti J, Chritchlow C, Corey L. Recurrences after oral and genital herpes simplex virus infection. N Engl J Med 1987;316:1444-9.

11 Van Griensven GJP, de Vroome EMM, Goudsmit J, Coutinho RA. Changes in sexual behaviour and the fall in incidence of HIV infection among homosexual men. $B r$ Med $J$ 1989; 298:218-21.

12 Van Griensven GJP, van den Hoek JAR, Leentvaar A, Coutinho RA. Surrogate markers for HIV incidence among homosexual men. $J$ Inf Dis 1989;159:1157-8.

Accepted for publication 2 July 1990 